\title{
Gas Turbine Blade Stress Analysis and Mode Shape Determination using Thermoelastic Methods \\ D. Backman ${ }^{1, a}$ and R.J. Greene ${ }^{2, b}$
}

${ }^{1}$ National Research Council Canada, Structures Materials and Propulsion Laboratory, Institute for Aerospace Research, M-14, 1200 Montreal Road, Ottawa, ON K1A 0R6, Canada

${ }^{2}$ The University of Sheffield, Dept Mechanical Engineering, Mappin Street, Sheffield S1 3JD, UK

adavid.backman@nrc-cnrc.gc.ca, 'r.j.greene@sheffield.ac.uk

Keywords: Thermoelasticity, differential infrared thermography, vibration, turbine blade.

\begin{abstract}
The efficacy of thermoelastic stress analysis for use in the study of moderately curved gas turbine blades is considered over a frequency range of $68 \mathrm{~Hz}$ to $3.4 \mathrm{kHz}$. A selection of blades, both industrial examples and simplified planar laboratory specimens, are excited at their natural vibration frequencies using both electromagnetic shakers and piezoelectric stack actuators, in order to develop a cyclic displacement of the blade surface and hence a cyclic variation in surface stress condition. Results are shown using both snapshot array and rolling array infrared detector systems, and the data then used to generate maps of normalized principal surface stress sum, and hence the mode shapes of vibration, including the first four excitation modes.
\end{abstract}

\section{Introduction}

Given the cyclic change in surface stress condition exhibited by most gas turbine blades when subjected to excitation at one of their natural vibration frequencies, these components are well suited to analysis using differential infrared thermography. The ability to experimentally obtain even a normalized map of the sum of principal stresses would be of great benefit in corroborating finite element models of blade behavior. The thermoelastic temperature change which occurs on the blade surface during excitation is related to the cyclic change in the sum of principal surface stresses by

$$
\Delta T=-\frac{\alpha T}{\rho C_{p}} \Delta\left(\sigma_{1}+\sigma_{2}+\sigma_{3}\right)
$$

where $T$ is the absolute temperature, $\alpha$ the coefficient of thermal expansion of the blade material, $\rho$ the density of the blade material, $C_{p}$ the specific heat at constant pressure, and $\sigma_{1}, \sigma_{2}$ and $\sigma_{3}$ are the principal stresses. Since a condition of plane stress will exist on the component surface, and since the material parameters in the above equation are approximately independent of temperature if $\Delta T$ is small, this can be reduced to

$$
A S=\Delta\left(\sigma_{1}+\sigma_{2}\right)
$$

where $A$ is a calibration constant and $S$ the magnitude of the thermoelastic response as recorded using an infrared detector. This well recognised result [1], in tandem with a suitable calibration, allows the surface distribution of principal stress sum to be determined from the component surface during excitation. However, the application of thermoelastic stress analysis methods to elevated frequency testing presents several particular experimental and image processing challenges which are rarely seen at the range of test frequencies achieved using servo-hydraulic test equipment.

Continuous temperature analysis. A number of research teams and industrial users have considered elevated frequency thermoelastic analysis and a modest range of results have been 
reported in the literature. The earliest published work was with the SPATE (Stress Pattern Analysis by Thermal Emission) thermoelastic analysis system, studying the response of gas turbine blade components at the frequency ranges of typical turbine blade free vibration $[2,3]$. This has since been followed up with further work using the SPATE detector system [4] and has demonstrated the effectiveness of this raster scanning system in the study of high frequency vibration analysis using the thermoelastic approach.

The SPATE detector and electronics within the optical head provide a continuous reading of the surface temperature of a single point on the surface of the component under test. This reading, output as a continuously varying voltage, is then passed to an analogue signal correlator which acts as a real-time filter on the time-varying infrared signal. If a reference signal is also provided to the correlator, for example from the sinusoidal input to the excitation hardware, the correlator then extracts only the magnitude of the oscillation of the infrared signal which varies at the same frequency as the reference signal. This analogue filtering process rejects all frequency components in the infrared signal which are not associated with the thermoelastic temperature variation on the blade surface and yields the magnitude of the thermoelastic response, and also its temporal relationship with the reference signal.

As a result of the continuously varying form of the infrared signal from this system, operation at elevated test frequencies is possible if the SPATE head electronics have a sufficiently fast response to changes in observed blade surface temperature, and if the correlator has a similar ability to accept and filter high frequency signals. The SPATE handbook [5] suggests an upper limit of $20 \mathrm{kHz}$ for the possible thermoelastic analysis frequency using the standard equipment configuration, although no experimental test results have been reported at anything approaching this frequency. The authors believe that this quoted limiting frequency is based on the capacity of the SPATE head and correlator electronics to correctly process a simulated infrared signal of this frequency, rather than a demonstrated ability to capture thermoelastic information in these conditions.

Temporally quantised temperature analysis. In contrast to the SPATE analysis described above, staring array infrared detectors are fundamentally different in their operation. Rather than a continuous signal which reports surface temperature from a point on the blade surface, staring array systems yield temporally quantised infrared data in the form of infrared images. These images are typically recorded during millisecond long periods for room temperature, servo-hydraulic test frame frequencies, and are then read out from the detector using a read out circuit (ROC) immediately behind the detector surface. For an infrared camera system capturing 200 of these infrared data images per second, or in other words a 200 frame per second (fps) camera system, the duration of each 'frame' is $1 / 200 \mathrm{~s}$ or $5 \mathrm{~ms}$. For a $10 \mathrm{~Hz}$ mechanical test, one complete loading cycle will last for $1 / 10 \mathrm{~s}$ or $100 \mathrm{~ms}$, and so 20 infrared images will be available for each loading cycle, enabling the temperature oscillation due to the thermoelastic response to be determined readily from the quantised infrared data.

It follows therefore that, as the test frequency increases, difficulties will begin to enter the analysis unless the frame rate of the infrared camera can be increased in line with the test frequency. The matter is further complicated by the availability of two differing constructions of staring array detector: snapshot array, where all the individual detector elements are recording simultaneously; and rolling array, where the individual detector elements are activated sequentially during each frame. The challenges in this staring array analysis, and the solutions employed by the authors during this initial research work, are described below.

\section{Elevated frequency challenges for thermoelastic analysis}

The following specific areas have been the focus of the present work and relate to the use of both snapshot and rolling array infrared detectors. As is clear from the description below, there are 
several parameters which work in concert to diminish the success of the thermoelastic analysis approach as the test frequency increases.

Image capture frequency. At test frequencies greater than approximately half of the infrared camera frame rate, care must be taken when selecting the camera frame rate. Perhaps counterintuitively, it is entirely possible to capture thermoelastic data with a test frequency which exceeds the camera frame rate, and this is demonstrated later in the paper.

There will clearly be a problem if the test frequency is the same or close to the camera frame rate, or one of its harmonics: in this situation each infrared data image will be identical, or nearly so, having been recorded from the same point in the loading cycle every time. This situation can be avoided by slowing the camera frame rate below the optimum frame rate for the system, which leads to a loss of efficiency in isolating the thermoelastic information and leads to an associated increase in the analysis time required, but regains the ability to isolate the thermoelastic result.

Another approach is to window the array or use binning, using a subset of the available detector elements, where the infrared hardware allows. Windowing simply excludes portions of the full data image from the camera head data stream, allowing a corresponding increase in camera frame rate since less data must be read out from the ROCs during each frame. Binning will typically cluster the array into a grid with groups of four, or nine, or 16 detector elements, and only interrogate one single detector within each group and return this result for all elements in the group. This effectively reduces the resolution of the camera detector chip and again reduces the data rate from each image frame, allowing an increase in frame rate.

However, neither of these camera frame rate increase approaches are immune from the limit of camera shutter speed (integration time).

Infrared camera shutter speed (integration time). The time period during which the individual detectors in the infrared array are sensitive to incident infrared photons during each camera frame is the camera integration time. The maximum integration time will be ultimately limited by the camera frame rate since the integration time must clearly not exceed the duration of each frame. However, once the test frequency approaches the camera frame rate, and certainly once it exceeds it, this limiting integration time does not lead to a successful analysis. Instead, an integration time of approximately one third of the test oscillation period allows for adequate sampling of the thermoelastic response, up to a few $\mathrm{kHz}$. Thus for a test frequency of $700 \mathrm{~Hz}$ as shown in a later example, each oscillation period will be $1 / 700 \mathrm{~s}$ or $1.4 \mathrm{~ms}$, with a camera integration time of approximately $0.5 \mathrm{~ms}$ yielding excellent results. As can be seen from the image capture frequency section above, for this $700 \mathrm{~Hz}$ example, increasing the camera frame rate by windowing or binning can only be done up to a point. Once the duration of each frame falls below the optimum camera integration time of $0.5 \mathrm{~ms}$, or a frame rate of $200 \mathrm{fps}$, there is no advantage to be gained by windowing or binning since this will lead to a loss of spatial data density without a corresponding increase in the quality of the thermoelastic data, or the rate at which it can be recorded.

Thermoelastic response magnitude. At lower test frequencies, turbine blades will oscillate in one of their first few natural modes of vibration, and it is relatively straightforward to induce substantial displacements and hence surface stress changes on the blade. As the test frequency increases, the blades will reach the higher modes of vibration and it becomes increasingly difficult to transmit sufficient power to the blade from the excitation system to induce significant displacements and surface stress oscillations.

Infrared signal attenuation. Unless the blade is being tested at elevated temperatures, it will usually be necessary to apply a uniformly radiative surface coating to the blade to obtain a consistent and high infrared emission. This in turn will reduce the magnitude of the temperature oscillation observed on the blade surface as the excitation frequency increases [6,7].

Data capture duration. All of the above issues lead to a tail chasing exercise at elevated test frequencies where a thermoelastic response of small magnitude is attenuated at the blade surface and then recorded using a short camera integration time with poor signal to noise ratio. The only 
method available to compensate for these unfavourable factors is to record a very large number of infrared data and use all these data to isolate and quantify the thermoelastic response. In practice this leads to long analysis times for staring array systems, from minutes in the range below $1 \mathrm{kHz}$ to hours in the higher $\mathrm{kHz}$ range.

\section{Excitation hardware}

Since the analysis of blade vibration when mounted in situ in an engine assembly by optical methods is impractical, the usual approach is to extract a single blade from the turbine and excite this blade in the laboratory. This not only gives precise control over the excitation frequency and magnitude, but also affords excellent visibility for the infrared camera systems in use. In most experimental cases, either electromagnetic shakers or piezoelectric actuators are employed, depending on the frequency of excitation required.

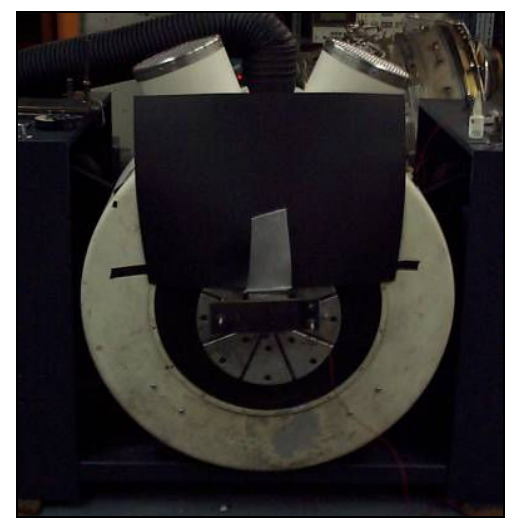

a)

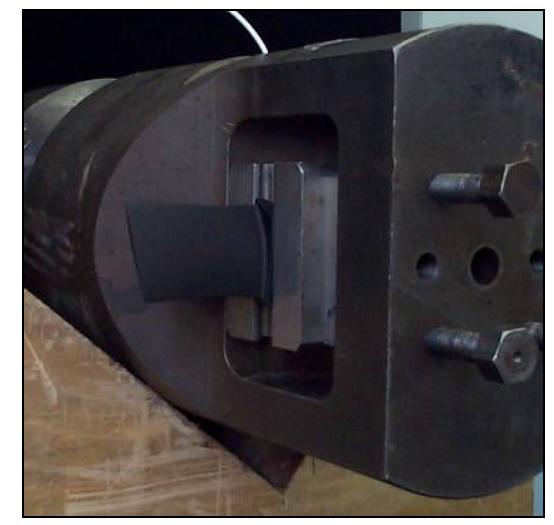

b)

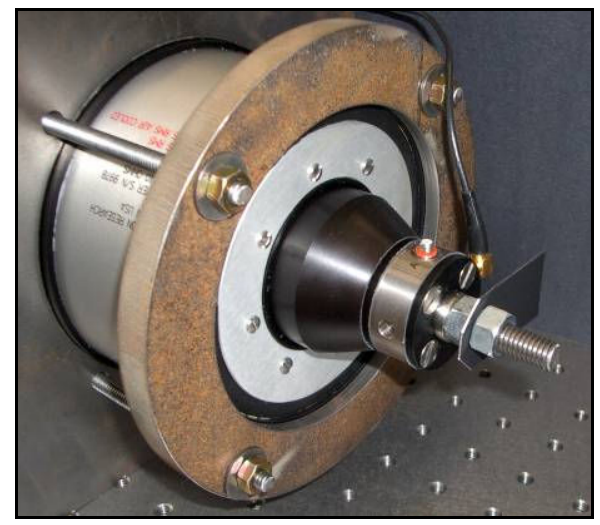

c)

Figure 1 - the three excitation actuators described, with different blades mounted for illustration: a) electromagnetic shaker, with mid-stage compressor blade; b) piezoelectric stack actuator, with turbine blade; c) combined electromagnetic/piezoelectric actuator, with planar blade.

Electromagnetic shakers. For low frequency excitation, typically less than $1 \mathrm{kHz}$, electromagnetic shaker systems offer excellent power and displacement for blade excitation. These systems are essentially the same in construction as music loud speakers, with a moving coil oscillating within a magnetic field. However, rather than sporting an external cone to convert the mechanical energy of the oscillation into sound energy, an electromagnetic shaker simply has a driving spigot at its centre which can then be linked to a blade assembly. An example is shown in Fig. 1a above.

Piezoelectric stack actuators. For high frequency excitation, typically greater than $1 \mathrm{kHz}$, electromagnetic shaker systems offer somewhat disappointing power and displacement - it is generally only possible to excite very low mass blades using these systems at the higher frequencies. An alternative approach, the piezoelectric stack, offers a solution with sufficient displacement at higher frequencies to transmit sufficient power into the blade in order to induce vibrations at the blade natural frequencies. Fig. $1 \mathrm{~b}$ shows a piezoelectric stack actuator, consisting of a horizontal sandwich of circular piezoelectric actuators within a steel casing.

Combined electromagnetic/piezoelectric actuators. Combining the above two technologies into a single actuator, using two separate sets of driving circuitry, allows a wide frequency range to be covered whilst transmitting sufficient power to blades of varying geometries. An example is shown in Fig. 1c.

\section{Results, rolling array detector}

Data captured using a rolling array detector (DeltaTherm 1410) has so far been restricted to laboratory specimens since the construction of a true blade excitation rig is still underway. The 
initial results shown here are from a series of aluminium simulated blades of thickness $0.5 \mathrm{~mm}$ and lengths from 150 to $30 \mathrm{~mm}$.

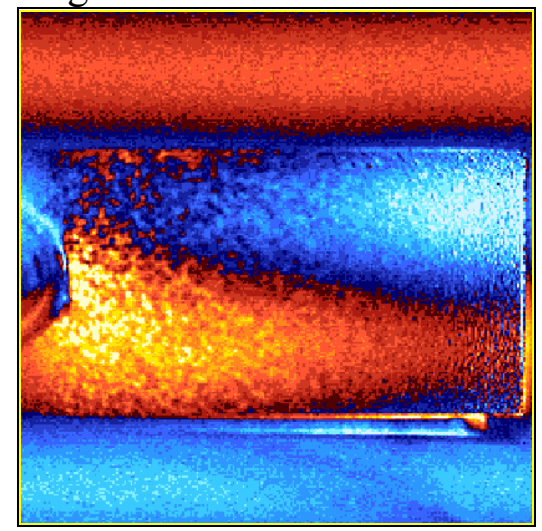

a)

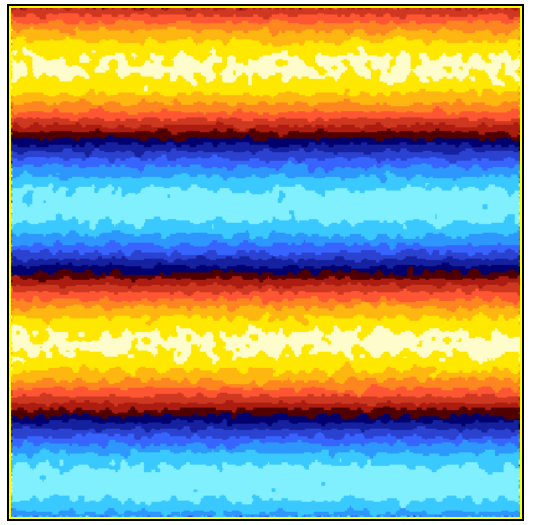

b)

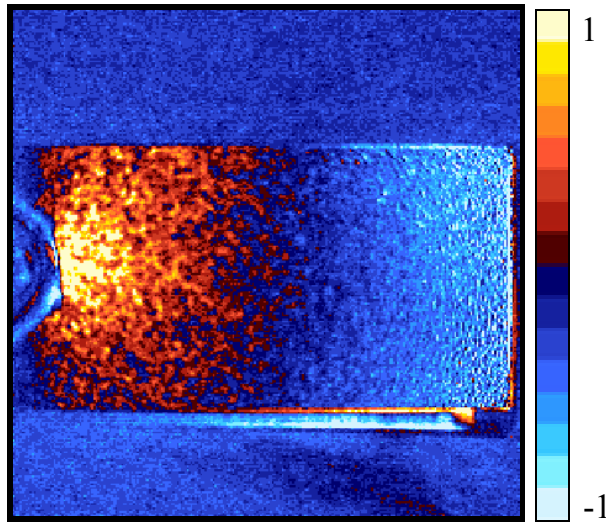

c)

Figure 2 - thermoelastic data from a $125 \mathrm{~mm}$ long planar laboratory specimen at first flap, $261 \mathrm{~Hz}$ : a) raw signal from the thermoelastic algorithm; b) correction signal, median filtered; c) thermoelastic data result from the subtraction of $b$ ) from a).

The raw data captured from the test appears as Fig. 2a, with the result integrated over 10 minutes. As is apparent from this figure, although the stress concentration at the blade root is visible within the distribution, there is a superimposed data field which forms horizontal banding within the image. This banding is due to a frequency dependent artefact generated as the infrared data is processed, and so a reference data image was captured without the specimen in the field of view of the camera. This null image, or reference image, is shown in Fig. 2b, where the periodic variation of the data in horizontal bands is clearly visible. The form of this reference data is sinusoidal along the vertical image axis, with maxima and minima at consistent magnitudes, and the low gradient of the data distribution was well suited to median filtering using a $3 \times 3$ pixel mask - the data in Fig. $2 b$ has undergone three median filter passes. Fig. $2 \mathrm{c}$ then shows the result of subtraction of the reference image from the raw data image, revealing the thermoelastic response of the blade.

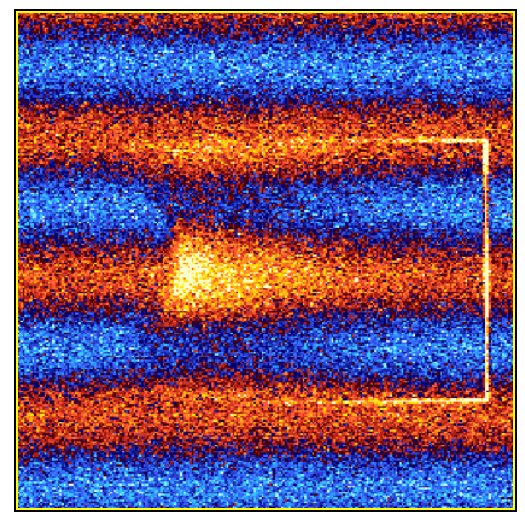

a)

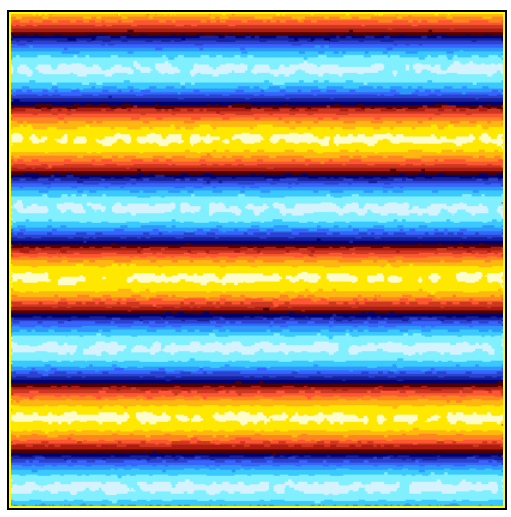

b)

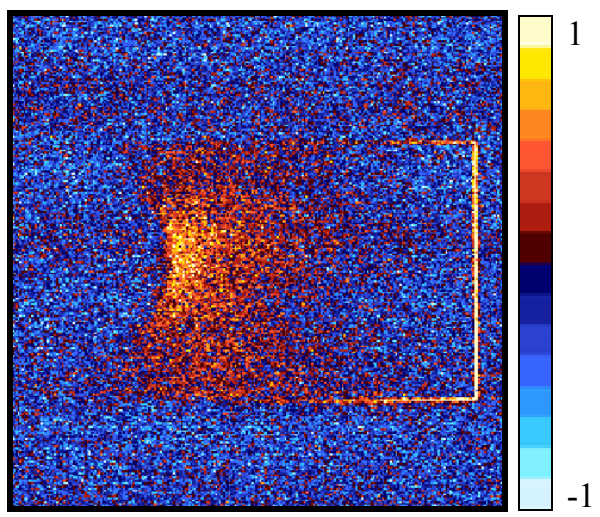

c)

Figure 3 - thermoelastic data from a $30 \mathrm{~mm}$ long planar laboratory specimen at first flap, $505 \mathrm{~Hz}$ : a) raw signal from the thermoelastic algorithm; b) correction signal, median filtered; c) thermoelastic data result from the subtraction of $b$ ) from $a$ ).

Fig. 3a shows the raw data captured from the test, integrating the result over 120 minutes. As can be seen by contrasting with Fig. 2b, Fig. $3 b$ reveals that the offset data field is strongly frequency dependent and so an offset image field must be recorded at each test frequency. Fig. $3 \mathrm{c}$ shows the subtraction of the reference image from the raw data and yields the thermoelastic response. It should be emphasised that the excitation magnitude of the blade during this test was barely detectable using a proximity probe, and the tip displacement was not visible to the eye. Thus the poor signal to noise 
ratio in the data in Fig. 3c is largely a product of a low magnitude cyclic change in stress condition.

\section{Results, snapshot array detector}

Two test data are presented, both from a DeltaTherm 1550 system: a $320 \mathrm{~mm}$ long compressor blade, showing the first four modes of vibration; and a $68 \mathrm{~mm}$ long turbine blade, showing the first two modes of vibration.

Compressor blade. Excited using an electromagnetic shaker, the first few natural frequencies of the blade were determined using a frequency sweep whilst monitoring the response of a tip-mounted single-axis accelerometer of $3 \mathrm{~mm}$ diameter. The blade was then excited at the first four vibration modes, shown in Fig. 4 below.

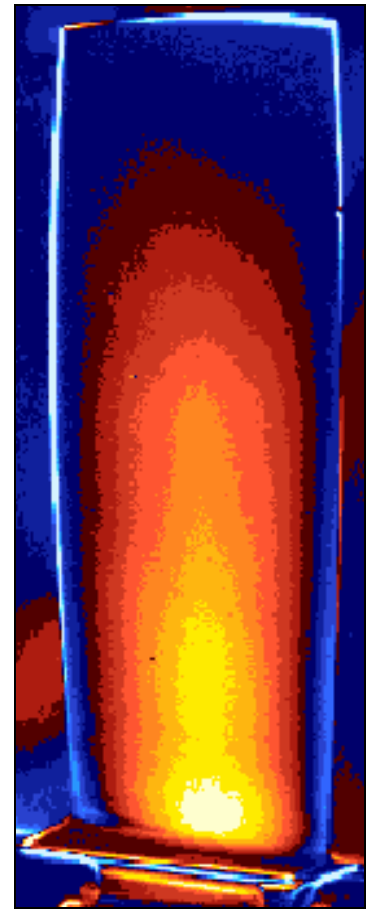

a)

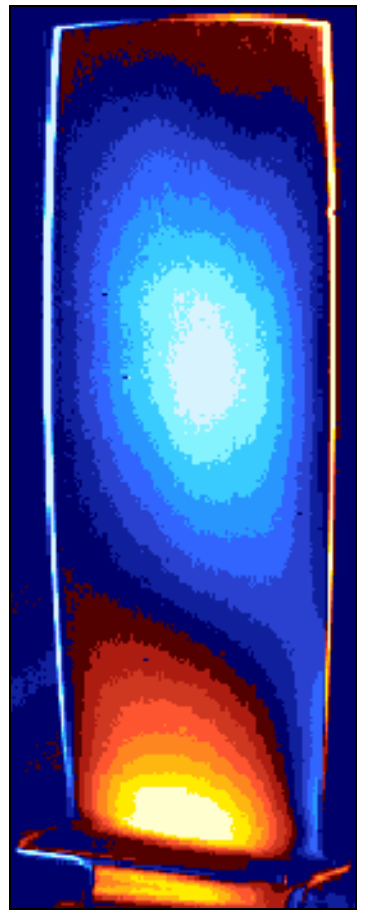

b)

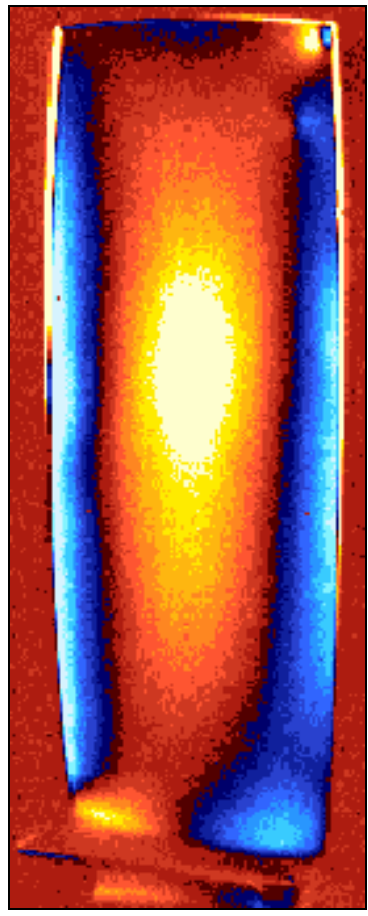

c)
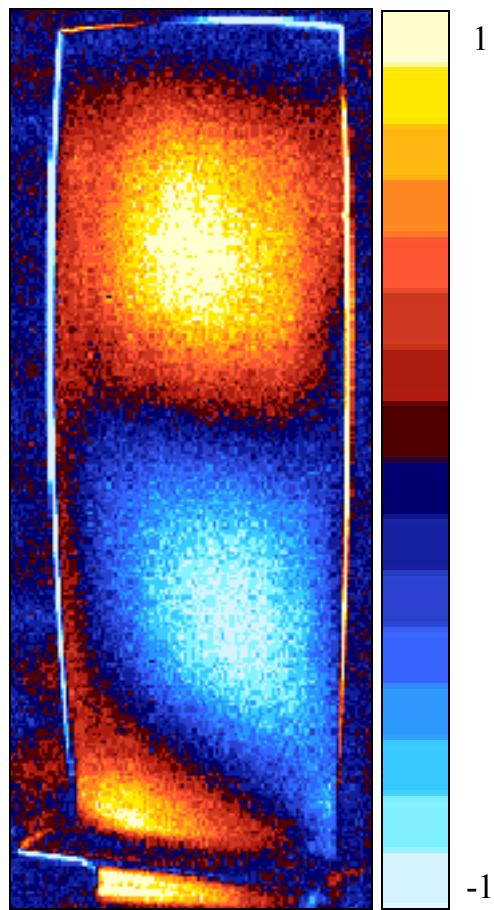

d)

Figure 4 - uncalibrated thermoelastic response of a $320 \mathrm{~mm}$ long compressor blade, normalised by the peak thermoelastic response: a) first flap $68 \mathrm{~Hz}$; b) second flap $237 \mathrm{~Hz}$; c) first torsion $433 \mathrm{~Hz}$; d) third flap $614 \mathrm{~Hz}$.

Each of the four data have been normalized by the peak response at the blade root and clearly reveal the first four vibrational modes: first flap, second flap, first torsion, and third flap.

Turbine blade. Excited using the piezoelectric stack actuator shown in Fig. 1b, the turbine blade in this study has a free length of $68 \mathrm{~mm}$ and is relatively untwisted along its length, presenting an almost flat surface for infrared observation during excitation. A resonance sweep was conducted as with the compressor blade above to identify the first two natural frequencies, which occurred at 701 $\mathrm{Hz}$ and $3.4 \mathrm{kHz}$. The blade was then excited using the piezoelectric stack actuator shown in Fig. $1 \mathrm{~b}$ at these two natural frequencies, the results of which are presented in Fig. 5, with the data normalized by the peak response at the blade root. Fig. 5a shows the response at $701 \mathrm{~Hz}$, the first natural frequency or first flap. Data has been integrated over 10 minutes and, as can be seen from the unfiltered data presented, the signal to noise ratio is excellent and the distribution of surface stresses is clear. Fig $5 \mathrm{~b}$ shows the response at $3.4 \mathrm{kHz}$, the second natural frequency or second flap. Data has been integrated over 30 minutes and although the macro-scale distribution is discernable within the image, correctly revealing the mode shape of vibration, there is a columnar variation in 
the data which is due to an unexpected processing artifact. This feature is the subject of current study.

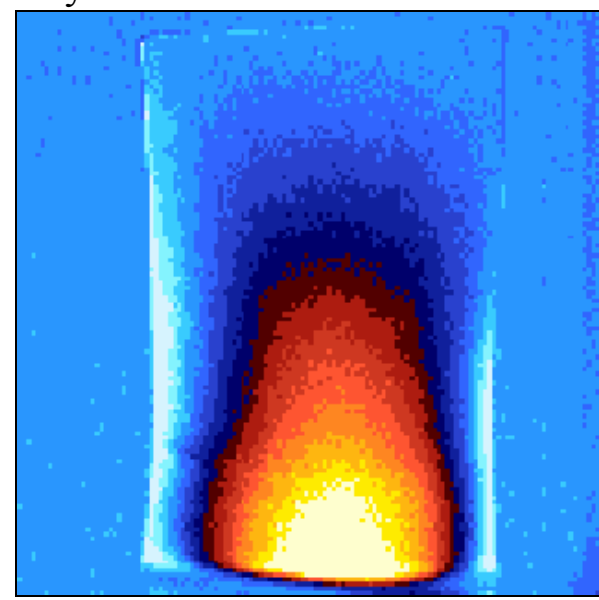

a)

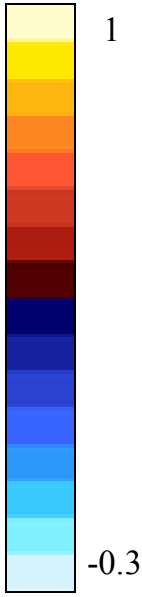

$-0.3$

Figure 5 - normalised thermoelastic response of a $68 \mathrm{~mm}$ long turbine blade: a) first flap 701

$\mathrm{Hz}$; b) second flap $3400 \mathrm{~Hz}$, with offset applied to alternating columns of data.

\section{Discussion and conclusions}

The data presented above demonstrates that thermoelastic assessment of turbine blade vibration is entirely viable using staring array detectors, both snapshot and rolling array. There are specific experimental challenges which are associated with elevated frequency analysis, both with the excitation method and the data capture approach, and these are the subject of ongoing development. Specifically, the intention is to parameterize the optimum data capture settings for the different infrared camera systems in order to develop an industrially robust test methodology.

In conclusion, initial data on the thermoelastic analysis of a variety of gas turbine blades at frequencies up to $3.4 \mathrm{kHz}$ has been presented, and modes of vibration up to and including the $4^{\text {th }}$ mode have been correctly identified using the recorded stress distributions. With the development of improved excitation hardware, future work will focus on the analysis of higher vibration modes in a bid to identify the maximum practical analysis frequencies for both rolling array and snapshot array infrared camera systems.

\section{Acknowledgements}

The authors wish to acknowledge the support of Marc Genest and Farid Mabrouki at NRC for the provision of the combined electromagnetic/piezoelectric shaker, Steve Turner and Ian Gower of Rolls-Royce plc for the provision of blades and excitation facilities, and The University of Sheffield for the use of thermoelastic test equipment and excitation hardware.

\section{References}

[1] G. Pitarresi and E.A. Patterson: J Strain Anal Eng Design 38 (5) (2003), pp. 405-417.

[2] S.R. Turner and N.G. Pollard: SPIE Vol. 713 Stress Analysis by Thermoelastic Techniques, $162-177,1987$.

[3] T.E. Purcell: Exptl Tech., 9-13, May/June 1996.

[4] R.A. Tomlinson, R.J. Greene and K. Delaney, in: Proc. SEM IX Int. Congress on Exptl Mech., June 5-8, Orlando, USA, 84-87, 2000.

[5] SPATE 4000 Series Operator's Manual, Issue 4, Ometron, (1996)

[6] J. McKelvie: SPIE 731, 44-53 (1987)

[7] A.K. Mackenzie: SPIE 1084, 59-71 (1989) 